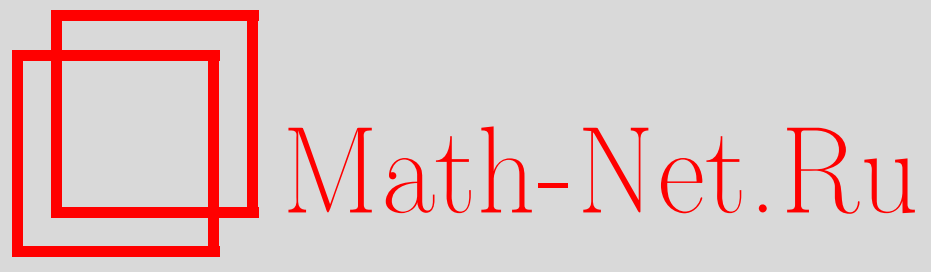

Г. У. Уразбоев, Цепочка Тоды со специальным самосогласованным источником, ТМФ, 2008, том 154, номер 2, 305-315

DOI: https://doi.org/10.4213/tmf6171

Использование Общероссийского математического портала Math-Net.Ru подразумевает, что вы прочитали и согласны с пользовательским соглашением http://www . mathnet.ru/rus/agreement

Параметры загрузки:

IP : 3.82 .47 .9

26 апреля 2023 г., 12:43:09

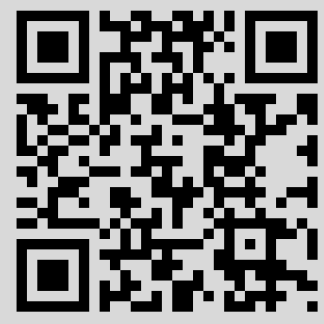




\section{ЦЕПОЧКА ТОДЫ СО СПЕЦИАЛЬНЫМ САМОСОГЛАСОВАННЫМ ИСТОЧНИКОМ}

Описывается метод интегрирования цепочки Тоды с самосогласованным источником с помощью метода обратной задачи рассеяния для дискретного оператора Штурма-Лиувилля с движущимися собственными значениями.

Ключевые слова: цепочка Тоды, самосогласованный источник, метод обратной задачи, движущиеся собственные значения.

\section{1. ВВЕДЕНИЕ}

В работе [1] была установлена важная роль, которую играют квадраты собственных функций в задачах на собственные значения для системы дифференциальных уравнений первого порядка. Ньюэлл показал [2], что именно квадраты собственных функций, а не сами собственные функции существенны при интегрировании с помощью метода обратной задачи для уравнения Штурма-Лиувилля. Строгое доказательство этого факта приведено в книге [3].

В данной работе рассматривается цепочка Тоды с самосогласованным источником

$$
\begin{aligned}
& \frac{d a_{n}}{d t}=a_{n}\left(b_{n}-b_{n+1}\right)+a_{n} \sum_{i=1}^{N}\left(f_{n+1}^{i} g_{n+1}^{i}-f_{n}^{i} g_{n}^{i}\right), \\
& \frac{d b_{n}}{d t}=2\left(a_{n-1}^{2}-a_{n}^{2}\right)+a_{n} \sum_{i=1}^{N}\left(f_{n}^{i} g_{n+1}^{i}+f_{n+1}^{i} g_{n}^{i}\right)-a_{n-1} \sum_{i=1}^{N}\left(f_{n}^{i} g_{n-1}^{i}+f_{n-1}^{i} g_{n}^{i}\right), \\
& a_{n-1} f_{n-1}^{k}+b_{n} f_{n}^{k}+a_{n} f_{n+1}^{k}=\lambda_{k} f_{n}^{k}, \\
& a_{n-1} g_{n-1}^{k}+b_{n} g_{n}^{k}+a_{n} g_{n+1}^{k}=\lambda_{k} g_{n}^{k}, \quad k=1,2, \ldots, N, \quad n \in \mathbb{Z},
\end{aligned}
$$

при начальном условии

$$
a_{n}(0)=a_{n}^{0}, \quad b_{n}(0)=b_{n}^{0}, \quad n \in \mathbb{Z} .
$$

Начальные данные $\left\{a_{n}^{0}\right\}_{-\infty}^{\infty},\left\{b_{n}^{0}\right\}_{-\infty}^{\infty}$ обладают следующими свойствами:

1) $a_{n}^{0}>0, \operatorname{Im} b_{n}^{0}=0, n \in \mathbb{Z}$;

*Ургенчский государственный университет, Ургенч, Узбекистан.

E-mail: gayrat71@mail.ru

4 Теоретическая и математическая физика, т. 154, № 2, 2008 г. 
2) $\sum_{n=-\infty}^{\infty}|n|^{3}\left(\left|a_{n}^{0}-1 / 2\right|+\left|b_{n}^{0}\right|\right)<\infty$;

3) дискретное уравнение Штурма-Лиувилля

$$
a_{n-1}^{0} y_{n-1}+b_{n}^{0} y_{n}+a_{n}^{0} y_{n+1}=\lambda y_{n}, \quad n \in \mathbb{Z},
$$

имеет ровно $N$ собственных значений $\lambda_{1}(0), \lambda_{2}(0), \ldots, \lambda_{N}(0)$, лежащих вне отрезка $[-1 ; 1]$.

В рассматриваемой задаче $\left\{a_{n}(t)\right\}_{-\infty}^{\infty},\left\{b_{n}(t)\right\}_{-\infty}^{\infty},\left\{f_{n}^{k}(t)\right\}_{-\infty}^{\infty},\left\{g_{n}^{k}(t)\right\}_{-\infty}^{\infty}, k=$ $1,2, \ldots, N,-$ неизвестные функции, причем $\left\{f_{n}^{k}(t)\right\}_{-\infty}^{\infty}$ есть собственный вектор оператора

$$
L(t) y \equiv a_{n-1} y_{n-1}+b_{n} y_{n}+a_{n} y_{n+1},
$$

соответствующий собственному значению $\lambda_{k}=\left(z_{k}+z_{k}^{-1}\right) / 2, k=1,2, \ldots, N$; функция $\left\{g_{n}^{k}(t)\right\}_{-\infty}^{\infty}$, являющаяся решением уравнения

$$
a_{n-1} g_{n-1}^{k}+b_{n} g_{n}^{k}+a_{n} g_{n+1}^{k}=\lambda_{k} g_{n}^{k}, \quad k=1,2, \ldots, N, \quad n \in \mathbb{Z},
$$

и собственный вектор $\left\{f_{n}^{k}(t)\right\}_{-\infty}^{\infty}$ линейно независимы.

Предполагается, что

$$
W\left\{f_{n}^{k}, g_{n}^{k}\right\} \equiv a_{n}\left(f_{n}^{k} g_{n+1}^{k}-f_{n+1}^{k} g_{n}^{k}\right)=\omega_{k}(t), \quad k=1,2, \ldots, N,
$$

где $\omega_{k}(t)$ - изначально заданные действительные и непрерывные функции от $t$, удовлетворяющие условию

$$
\left|\frac{\lambda_{k}(0)}{2}+\int_{0}^{t} \omega_{k}(t) d t\right|>\frac{1}{2}, \quad k=1,2, \ldots, N,
$$

при всех неотрицательных значениях $t$.

Решение задачи (1.1)-(1.4) ищется в классе функций $a_{n}(t), b_{n}(t)$, принадлежащих $C^{1}[0, \infty)$ и удовлетворяющих условиям

$$
a_{n}(t)>0, \quad \operatorname{Im} b_{n}=0, \quad n \in \mathbb{Z}, \quad \sup _{0 \leqslant t \leqslant T} \sum_{n=-\infty}^{\infty}|n|\left(\left|a_{n}(t)-\frac{1}{2}\right|+\left|b_{n}(t)\right|\right)<\infty
$$

при любом $T>0$.

Основная цель данной работы - получить представления для решений $\left\{a_{n}(t)\right\}_{-\infty}^{\infty}$, $\left\{b_{n}(t)\right\}_{-\infty}^{\infty},\left\{f_{n}^{k}(t)\right\}_{-\infty}^{\infty},\left\{g_{n}^{k}(t)\right\}_{-\infty}^{\infty}, k=1,2, \ldots, N$, задачи (1.1)-(1.5) в рамках метода обратной задачи рассеяния для оператора $L(t)$.

В работах [4]-[6] было показано, что цепочка Тоды без источника

$$
\begin{aligned}
& \dot{a}_{n}=a_{n}\left(b_{n}-b_{n+1}\right), \\
& \dot{b}_{n}=2\left(a_{n-1}^{2}-a_{n}^{2}\right), \quad n \in \mathbb{Z},
\end{aligned}
$$

может быть проинтегрирована методом обратной задачи рассеяния для разностного оператора Штурма-Лиувилля $L(t)$.

В связи с рассматриваемой задачей следует отметить работы [7], [8], в которых рассмотрены уравнения Кортевега-де Фриза с самосогласованным источником в связи с применением метода обратной задачи рассеяния для оператора ШтурмаЛиувилля. 


\section{2. НЕОБХОДИМЫЕ СВЕДЕНИЯ ИЗ ТЕОРИИ РАССЕЯНИЯ}

Рассмотрим разностное уравнение второго порядка

$$
L y \equiv a_{n-1} y_{n-1}+b_{n} y_{n}+a_{n} y_{n+1}=\lambda y_{n}, \quad n \in \mathbb{Z},
$$

где $\left\{y_{n}\right\}_{-\infty}^{\infty}-$ искомое решение, $\lambda=\left(z+z^{-1}\right) / 2-$ спектральный параметр и

$$
a_{n}>0, \quad \operatorname{Im} b_{n}=0, \quad n \in \mathbb{Z}, \quad \sum_{n=-\infty}^{\infty}|n|\left(\left|a_{n}-\frac{1}{2}\right|+\left|b_{n}\right|\right)<\infty .
$$

В этом разделе будут приведены хорошо известные, но необходимые для дальнейшего сведения, касающиеся теории прямой и обратной задач рассеяния для уравнения (2.1). Эта теория была развита в работе [9]; необходимые и достаточные условия разрешимости обратной задачи рассеяния были получены в работе [10]. Здесь мы будем придерживаться обозначений, принятых в монографии [11].

При условиях (2.2) решения Йоста уравнения (2.1) определяются асимптотиками

$$
\begin{array}{lll}
\varphi_{n}(z)=z^{n}+o(1), & n \rightarrow \infty, & |z|=1, \\
\psi_{n}(z)=z^{-n}+o(1), & n \rightarrow-\infty, & |z|=1 .
\end{array}
$$

Такие решения существуют, определяются асимптотиками (2.3) однозначно и аналитически продолжаются во внутреннюю часть круга $|z|<1$.

Для функции $\varphi_{n}(z)$ имеет место представление

$$
\varphi_{n}(z)=\sum_{n^{\prime}=n}^{\infty} K\left(n, n^{\prime}\right) z^{n^{\prime}}
$$

с коэффициентами $K\left(n, n^{\prime}\right)$, не зависящими от $z$ и связанными с $a_{n}$ и $b_{n}$ посредством равенств

$$
a_{n}=\frac{1}{2} \frac{K(n+1, n+1)}{K(n, n)}, \quad b_{n}=\frac{1}{2}\left(\frac{K(n, n+1)}{K(n, n)}-\frac{K(n-1, n)}{K(n-1, n-1)}\right) .
$$

При $|z|=1$ пары $\left\{\varphi_{n}(z), \varphi_{n}\left(z^{-1}\right)\right\}$ и $\left\{\psi_{n}(z), \psi_{n}\left(z^{-1}\right)\right\}$ образуют фундаментальную систему решений уравнения (2.1), поэтому

$$
\psi_{n}(z)=\alpha(z) \varphi_{n}\left(z^{-1}\right)+\beta(z) \varphi_{n}(z) .
$$

Заметим, что

$$
\alpha(z)=\frac{2}{z-z^{-1}} W\left\{\psi_{n}(z), \varphi_{n}(z)\right\} .
$$

Функция $\alpha(z)$ аналитически продолжается во внутреннюю часть круга $|z|<1$ и имеет там конечное число простых нулей $z_{1}, z_{2}, \ldots, z_{N}$, причем $\lambda_{k}=\left(z_{k}+z_{k}^{-1}\right) / 2$, $k=1,2, \ldots, N$, есть собственное значение оператора $L$. Из формулы (2.7) следует, что

$$
\psi_{n}^{k}=\beta_{k} \varphi_{n}^{k}, \quad k=1,2, \ldots, N
$$


где $\psi_{n}^{k}=\psi_{n}\left(z_{k}\right)$.

Если $\lambda_{k}=\left(z_{k}+z_{k}^{-1}\right) / 2$ - собственное значение оператора $L$, то нормированным собственным вектором, соответствующим этому собственному значению, мы будем называть собственный вектор $\left\{\xi_{n}^{k}\right\}_{n=-\infty}^{\infty}$, удовлетворяющий условию

$$
\sum_{n=-\infty}^{\infty}\left(\xi_{n}^{k}\right)^{2}=1
$$

Ясно, что $\xi_{n}^{k}=C_{k} \varphi_{n}^{k}, k=1,2, \ldots, N, n \in \mathbb{Z}$. Заметим, что в силу хорошо известного равенства

$$
\dot{\alpha}\left(z_{k}\right)=-\frac{1}{z_{k}} \sum_{j=-\infty}^{\infty} \varphi_{j}\left(z_{k}\right) \psi_{j}\left(z_{k}\right)
$$

мы имеем

$$
C_{k}^{2}=-\frac{\beta_{k}}{z_{k} \dot{\alpha}\left(z_{k}\right)}, \quad k=1,2, \ldots, N .
$$

Функция $R(z)=\beta(z) / \alpha(z)$ называется коэффициентом отражения. Гладкая на окружности $|z|=1$, эта функция удовлетворяет инволюции

$$
\bar{R}(z)=R(\bar{z})
$$

и условию

$$
|R(z)| \leqslant 1
$$

причем знак равенства может достигаться лишь в точках $z= \pm 1$.

Для аналитического продолжения функции $\alpha(z)$ во внутреннюю часть круга $|z|<1$ необходимо выполнение равенства

$$
\alpha(0)=\prod_{j=1}^{N}\left|z_{j}\right| \exp \left\{\frac{i}{4 \pi} \int_{|\zeta|=1} \ln \left(1-|R(\zeta)|^{2}\right) \frac{d \zeta}{\zeta}\right\} .
$$

Набор $\left\{R(z), z_{1}, z_{2}, \ldots, z_{N}, C_{1}, C_{2}, \ldots, C_{N}\right\}$ называется данными рассеяния для уравнения (2.1).

Коэффициенты в представлении (2.4) являются решением уравнений ГельфандаЛевитана-Марченко

$$
\begin{gathered}
\chi(n, m)+F(n+m)+\sum_{n^{\prime}=n+1}^{\infty} \chi\left(n, n^{\prime}\right) F\left(n^{\prime}+m\right)=0, \quad m>n, \\
(K(n, n))^{-2}=1+F(2 n)+\sum_{n^{\prime}=n+1}^{\infty} \chi\left(n, n^{\prime}\right) F\left(n^{\prime}+n\right),
\end{gathered}
$$

где

$$
\begin{gathered}
F(n)=\frac{1}{2 \pi i} \oint_{|z|=1} R(z) z^{n-1} d z+\sum_{k=1}^{N} C_{k}^{2} z_{k}^{n}, \\
\chi(n, m)=\frac{K(n, m)}{K(n, n)} .
\end{gathered}
$$


Если попарно не равные числа $z_{k} \neq 0$ лежат в интервале $-1<z_{k}<1, k=$ $1,2, \ldots, N$, а функция $R(z)$ на окружности $|z|=1$ обладает свойствами $(2.10),(2.11)$ и справедливо условие (2.12), то уравнение Гельфанда-Левитана-Марченко (2.13) однозначно разрешимо (см. книгу [12]).

Теперь $\left\{a_{n}\right\}_{-\infty}^{\infty},\left\{b_{n}\right\}_{-\infty}^{\infty}$ определяются с помощью данных рассеяния из (2.5).

Заметим, что вектор

$$
h_{n}^{k}=\left.\frac{d}{d z}\left(\psi_{n}(z)-\beta_{k} \varphi_{n}(z)\right)\right|_{z=z_{k}}
$$

является решением уравнения $L y=\lambda_{k} y, k=1,2, \ldots, N$.

Так как из равенства $(2.7)$ при $|z|<1$ следует, что $\varphi_{n}(z) \rightarrow \alpha(z) z^{n}$ при $\quad n \rightarrow-\infty$, то $h_{n}^{k} \rightarrow-\beta_{k} \dot{\alpha}\left(z_{k}\right) z_{k}^{n}$ при $n \rightarrow-\infty, k=1,2, \ldots, N$. Следовательно, из асимптотик (2.3) получаем

$$
W\left\{h_{n}^{k}, \psi_{n}^{k}\right\}=\frac{\beta_{k} \dot{\alpha}\left(z_{k}\right)\left(z_{k}-z_{k}^{-1}\right)}{2} .
$$

Справедлива следующая лемма.

Лемма 1. Пусть $\left\{x_{n}(\lambda)\right\}_{-\infty}^{\infty},\left\{y_{n}(\mu)\right\}_{-\infty}^{\infty}$-решения уравнений $L x=\lambda x$ и $L y=$ $\mu$, соответственно. Тогда имеет место тождество

$$
(\mu-\lambda) x_{n}(\lambda) y_{n}(\mu)=W\left\{x_{n}(\lambda), y_{n}(\mu)\right\}-W\left\{x_{n-1}(\lambda), y_{n-1}(\mu)\right\}, \quad n \in \mathbb{Z} .
$$

Лемма доказывается непосредственной проверкой.

Из этой леммы, в частности, следует независимость от $n$ вронскианов двух решений уравнений (2.1) при одинаковых спектральных параметрах и ортогональность собственных векторов, соответствующих различным собственным значениям.

\section{3. ЭВОЛЮЦИЯ ДАННЫХ РАССЕЯНИЯ}

Пусть $\left\{\Phi_{n}^{k}(t)\right\}_{-\infty}^{\infty}-$ нормированный собственный вектор оператора $L(t)$, соответствующий собственному значению $\lambda_{k}(t), k=1,2, \ldots, N$, т.е.

$$
a_{n-1} \Phi_{n-1}^{k}+b_{n} \Phi_{n}^{k}+a_{n} \Phi_{n+1}^{k}=\lambda_{k} \Phi_{n}^{k}, \quad n \in \mathbb{Z}
$$

Продифференцировав эти равенства по $t$ и умножив затем на $\Phi_{n}^{k}$, просуммируем по $n$ от $-\infty$ до $\infty$. Используя уравнения (1.1) и самосопряженность оператора $L(t)$, получим

$$
\begin{gathered}
\frac{d \lambda_{k}}{d t}=\sum_{i=1}^{N} \sum_{n=-\infty}^{\infty}\left(a_{n-1}\left(f_{n}^{i} g_{n}^{i}-f_{n-1}^{i} g_{n-1}^{i}\right) \Phi_{n-1}^{k} \Phi_{n}^{k}+a_{n}\left(f_{n}^{i} g_{n+1}^{i}+f_{n+1}^{i} g_{n}^{i}\right)\left(\Phi_{n}^{k}\right)^{2}-\right. \\
\left.-a_{n-1}\left(f_{n}^{i} g_{n-1}^{i}+f_{n-1}^{i} g_{n}^{i}\right)\left(\Phi_{n}^{k}\right)^{2}+a_{n}\left(f_{n+1}^{i} g_{n+1}^{i}-f_{n}^{i} g_{n}^{i}\right) \Phi_{n+1}^{k} \Phi_{n}^{k}\right) .
\end{gathered}
$$

Последнее равенство можно переписать в виде

$$
\frac{d \lambda_{k}}{d t}=\sum_{i=1}^{N} \sum_{n=-\infty}^{\infty}\left[2 a_{n}\left(f_{n+1}^{i} g_{n+1}^{i}-f_{n}^{i} g_{n}^{i}\right) \Phi_{n+1}^{k} \Phi_{n}^{k}+a_{n}\left(f_{n}^{i} g_{n+1}^{i}+f_{n+1}^{i} g_{n}^{i}\right)\left(\left(\Phi_{n}^{k}\right)^{2}-\left(\Phi_{n+1}^{k}\right)^{2}\right] .\right.
$$


Группируя слагаемые, получим

$$
\begin{aligned}
\frac{d \lambda_{k}}{d t}=\sum_{i=1}^{N} \sum_{n=-\infty}^{\infty}\left[f_{n+1}^{i} \Phi_{n+1}^{k} W\left\{\Phi_{n}^{k}, g_{n}^{i}\right\}+g_{n+1}^{i} \Phi_{n+1}^{k} W\left\{\Phi_{n}^{k}, f_{n}^{i}\right\}+\right. \\
\left.\quad+f_{n}^{i} \Phi_{n}^{k} W\left\{\Phi_{n}^{k}, g_{n}^{i}\right\}+g_{n}^{i} \Phi_{n}^{k} W\left\{\Phi_{n}^{k}, f_{n}^{i}\right\}\right] .
\end{aligned}
$$

ЛЕмма 2. При $i \neq k$ выполняется равенство

$$
\sum_{n=-\infty}^{\infty}\left[W\left\{\Phi_{n}^{k}, g_{n}^{i}\right\}\left(f_{n+1}^{i} \Phi_{n+1}^{k}+f_{n}^{i} \Phi_{n}^{k}\right)+W\left\{\Phi_{n}^{k}, f_{n}^{i}\right\}\left(g_{n+1}^{i} \Phi_{n+1}^{k}+g_{n}^{i} \Phi_{n}^{k}\right)\right]=0 .
$$

ДокАЗАтЕЛьСтво. Для удобства введем обозначения $W_{n}=W\left\{\Phi_{n}^{k}, g_{n}^{i}\right\}, V_{n}=$ $W\left\{\Phi_{n}^{k}, f_{n}^{i}\right\}$. Используя лемму 1 , получим

$$
\begin{aligned}
& \sum_{n=-\infty}^{\infty}\left[W_{n}\left(f_{n+1}^{i} \Phi_{n+1}^{k}+f_{n}^{i} \Phi_{n}^{k}\right)+V_{n}\left(g_{n+1}^{i} \Phi_{n+1}^{k}+g_{n}^{i} \Phi_{n}^{k}\right)\right]= \\
& =\sum_{n=-\infty}^{\infty}\left[f_{n}^{i} \Phi_{n}^{k}\left(W_{n}+W_{n-1}\right)+g_{n}^{i} \Phi_{n}^{k}\left(V_{n}+V_{n-1}\right)\right]= \\
& =\frac{1}{\lambda_{i}-\lambda_{k}} \sum_{n=-\infty}^{\infty}\left[\left(V_{n}-V_{n-1}\right)\left(W_{n}+W_{n-1}\right)+\left(W_{n}-W_{n-1}\right)\left(V_{n}+V_{n-1}\right)\right]= \\
& =\frac{2}{\lambda_{i}-\lambda_{k}} \sum_{n=-\infty}^{\infty}\left(W_{n} V_{n}-W_{n-1} V_{n-1}\right)=0,
\end{aligned}
$$

что и требовалось доказать.

Так как $W\left\{\Phi_{n}^{k}, f_{n}^{k}\right\}=0$, в силу формулы (1.3) и леммы 2 равенство (3.1) примет вид

$$
\frac{d \lambda_{k}}{d t}=2 \omega_{k}(t), \quad k=1,2, \ldots, N
$$

Рассмотрим систему уравнений

$$
\begin{gathered}
(L y)_{n} \equiv a_{n-1} y_{n-1}+b_{n} y_{n}+a_{n} y_{n+1}=\lambda y_{n}, \quad n \in \mathbb{Z}, \\
p_{n+1}^{k}-p_{n}^{k}=f_{n+1}^{k} y_{n+1}+f_{n}^{k} y_{n}, \quad k=1,2, \ldots, N,
\end{gathered}
$$

относительно неизвестных вектор-функций $\left\{p_{n}^{k}(z, t)\right\}, k=1,2, \ldots, N$. С помощью произвольного решения этой системы определим

$$
S_{n}^{0}=\frac{\partial y_{n}}{\partial t}+a_{n} y_{n+1}-a_{n-1} y_{n-1}+\sum_{m=1}^{N} g_{n}^{m} p_{n}^{m},
$$

$S_{n}^{k}=a_{n}\left(f_{n+1}^{k} y_{n}-f_{n}^{k} y_{n+1}\right)+a_{n-1}\left(f_{n}^{k} y_{n-1}-f_{n-1}^{k} y_{n}\right)+\left(\lambda-\lambda_{k}\right) p_{n}^{k}, \quad k=1,2, \ldots, N$.

Непосредственной проверкой можно показать, что

$$
S_{n}^{k}-S_{n-1}^{k}=0, \quad k=1,2, \ldots, N, \quad n \in \mathbb{Z},
$$


т.е. величины $S_{n}^{k}$ не зависят от $n$ при $k=1,2, \ldots, N$. Кроме того, из равенств (1.1), (3.3) и (3.5) следует, что

$$
(L-\lambda) S_{n}^{0}=-\sum_{m=1}^{N} g_{n}^{m} S_{n}^{m}, \quad n \in \mathbb{Z} .
$$

Пусть $\varphi_{n}(z, t)$ и $\psi_{n}(z, t)$ - решения Йоста уравнения (3.3) с асимптотиками (2.3). Полагая в $(3.4) y_{n}^{+} \equiv \varphi_{n}(z), y_{n}^{-} \equiv \psi_{n}(z)$, определим

$$
\begin{aligned}
& p_{n}^{k-}(z)=f_{n}^{k} \psi_{n}(z)+2 \sum_{j=-\infty}^{n-1} f_{j}^{k} \psi_{j}(z), \\
& p_{n}^{k+}(z)=-f_{n}^{k} \varphi_{n}(z)-2 \sum_{j=n+1}^{\infty} f_{j}^{k} \varphi_{j}(z), \quad k=1,2, \ldots, N .
\end{aligned}
$$

$\mathrm{C}$ помощью $y_{n}^{+} \equiv \varphi_{n}(z), y_{n}^{-} \equiv \psi_{n}(z), p_{n}^{1+}, p_{n}^{1-}, p_{n}^{2+}, p_{n}^{2-}, \ldots, p_{n}^{N+}, p_{n}^{N-}$ можно по формулам (3.5) и (3.6) определить $S_{n}^{i+}, S_{n}^{i-}, i=0,1, \ldots, N$.

На основании (3.4) и (3.6) можно утвержать, что $S_{n}^{i+} \underset{n \rightarrow \infty}{\longrightarrow} 0, S_{n}^{i-} \underset{n \rightarrow-\infty}{\longrightarrow} 0, i=$ $1, \ldots, N$. Поэтому из равенств $(3.7)$ следует, что

$$
S_{n}^{i+}=S_{n}^{i-}=0, \quad i=1, \ldots, N, \quad n \in \mathbb{Z} .
$$

Подставляя (3.10) в (3.8), получим

$$
(L-\lambda) S_{n}^{0+}=(L-\lambda) S_{n}^{0-}=0 .
$$

Так как $\left\{f_{n}^{k}(t)\right\}_{-\infty}^{\infty}$ есть собственный вектор оператора $L(t)$, соответствующий собственному значению $\lambda_{k}=\left(z_{k}+z_{k}^{-1}\right) / 2$, то

$$
f_{n}^{k}=c_{k}^{-} \psi_{n}\left(z_{k}, t\right)=c_{k}^{+} \varphi_{n}\left(z_{k}, t\right),
$$

т.е. $c_{k}^{+}=\beta_{k} c_{k}^{-}, k=1,2, \ldots, N$.

Согласно формулам (1.3) и (3.12)

$$
g_{n}^{k} \underset{n \rightarrow \infty}{\sim}-\frac{2 \omega_{k}}{c_{k}^{+}\left(z_{k}-z_{k}^{-1}\right)} z_{k}^{-n}, \quad g_{n}^{k} \underset{n \rightarrow-\infty}{\sim} \frac{2 \omega_{k}}{c_{k}^{-}\left(z_{k}-z_{k}^{-1}\right)} z_{k}^{n}, \quad k=1,2, \ldots, N .
$$

На основании формул $(3.4),(3.12)$ и асимптотик (2.3) получаем

$$
\begin{gathered}
p_{n}^{k-} \underset{n \rightarrow-\infty}{\sim} \frac{c_{k}^{-}\left(1+z_{k} z\right)}{1-z_{k} z}\left(z_{k} z\right)^{-n}, \quad p_{n}^{k+} \underset{n \rightarrow \infty}{\sim}-\frac{c_{k}^{+}\left(1+z_{k} z\right)}{1-z_{k} z}\left(z_{k} z\right)^{n}, \\
k=1,2, \ldots, N .
\end{gathered}
$$

Поэтому вследствие (1.5), (3.5) и (3.13) можно записать

$$
\begin{aligned}
& S_{n}^{0-} \underset{n \rightarrow-\infty}{\sim}\left(\frac{z^{-1}-z}{2}+2 \sum_{m=1}^{N} \frac{\left(1+z_{m} z\right) \omega_{m}}{\left(1-z_{m} z\right)\left(z_{m}-z_{m}^{-1}\right)}\right) z^{-n}, \\
& S_{n}^{0+} \underset{n \rightarrow \infty}{\sim}\left(\frac{z-z^{-1}}{2}+2 \sum_{m=1}^{N} \frac{\left(1+z_{m} z\right) \omega_{m}}{\left(1-z_{m} z\right)\left(z_{m}-z_{m}^{-1}\right)}\right) z^{n} .
\end{aligned}
$$


Следовательно, согласно равенству (3.11)

$$
\begin{aligned}
& S_{n}^{0-}=\left(\frac{z^{-1}-z}{2}+2 \sum_{m=1}^{N} \frac{\left(1+z_{m} z\right) \omega_{m}}{\left(1-z_{m} z\right)\left(z_{m}-z_{m}^{-1}\right)}\right) \psi_{n}(z), \\
& S_{n}^{0+}=\left(\frac{z-z^{-1}}{2}+2 \sum_{m=1}^{N} \frac{\left(1+z_{m} z\right) \omega_{m}}{\left(1-z_{m} z\right)\left(z_{m}-z_{m}^{-1}\right)}\right) \varphi_{n}(z) .
\end{aligned}
$$

Определим величину $G_{0}$ следующим образом:

$$
G_{0}=S_{n}^{0-}(z)-\alpha(z) S_{n}^{0+}\left(z^{-1}\right)-\beta(z) S_{n}^{0+}(z) .
$$

Согласно формулам (2.6) и (3.14)

$$
G_{0}=2 \sum_{m=1}^{N}\left(\frac{1+z_{m} z}{1-z_{m} z}-\frac{1+z_{m} z^{-1}}{1-z_{m} z^{-1}}\right) \frac{\omega_{m}}{z_{m}-z_{m}^{-1}} \alpha(z) \varphi_{n}\left(z^{-1}\right)+\left(z^{-1}-z\right) \beta(z) \varphi_{n}(z) .
$$

С другой стороны, из формул (2.6) и (3.5) следует, что

$$
\begin{gathered}
S_{n}^{0-}(z)=\frac{\partial \alpha(z)}{\partial t} \varphi_{n}\left(z^{-1}\right)+\frac{\partial \beta(z)}{\partial t} \varphi_{n}(z)+\alpha(z) S_{n}^{0+}\left(z^{-1}\right)+\beta(z) S_{n}^{0+}(z)+ \\
+\sum_{m=1}^{N} g_{n}^{m}\left(p_{n}^{m-}(z)-\alpha(z) p_{n}^{m+}\left(z^{-1}\right)-\beta(z) p_{n}^{m+}(z)\right) .
\end{gathered}
$$

Используя формулы (2.6), (3.9) и лемму 1, имеем

$$
p_{n}^{m-}(z)-\alpha(z) p_{n}^{m+}\left(z^{-1}\right)-\beta(z) p_{n}^{m+}(z)=2 \sum_{j=-\infty}^{\infty} f_{j}^{m} \psi_{j}(z)=0 .
$$

Следовательно, из определения $G_{0}$ и равенства (3.16) получаем

$$
G_{0}=\frac{\partial \alpha(z)}{\partial t} \varphi_{n}\left(z^{-1}\right)+\frac{\partial \beta(z)}{\partial t} \varphi_{n}(z) .
$$

Сравнивая (3.15) и (3.17), имеем

$$
\begin{gathered}
\frac{\partial \alpha(z)}{\partial t}=2 \sum_{m=1}^{N}\left(\frac{1+z_{m} z}{1-z_{m} z}-\frac{1+z_{m} z^{-1}}{1-z_{m} z^{-1}}\right) \frac{\omega_{m}}{z_{m}-z_{m}^{-1}} \alpha(z), \\
\frac{\partial \beta(z)}{\partial t}=\left(z^{-1}-z\right) \beta(z), \quad|z|=1 .
\end{gathered}
$$

Таким образом,

$$
R(z, t)=R(z, 0) e^{\theta(z, t)},
$$

где

$$
\theta(z, t)=\left(z^{-1}-z\right) t-2 \sum_{m=1}^{N} \int_{0}^{t}\left(\frac{1+z_{m}(\tau) z}{1-z_{m}(\tau) z}-\frac{1+z_{m}(\tau) z^{-1}}{1-z_{m}(\tau) z^{-1}}\right) \frac{\omega_{m}(\tau)}{z_{m}(\tau)-z_{m}^{-1}(\tau)} d \tau
$$


Положим

$$
G_{k}=S_{n}^{0-}\left(z_{k}\right)-\beta_{k} S_{n}^{0+}\left(z_{k}\right), \quad k=1,2, \ldots, N .
$$

Тогда на основании формул (2.8) и (3.14) получаем

$$
G_{k}=\left(z_{k}^{-1}-z_{k}\right) \beta_{k} \varphi_{n}\left(z_{k}\right), \quad k=1,2, \ldots, N .
$$

Дифференцируя равенство (2.8) по $t$ с учетом определения вектора $h_{n}^{k}$, приходим к

$$
\frac{\partial \psi_{n}\left(z_{k}\right)}{\partial t}=\frac{d \beta_{k}}{d t} \varphi_{n}\left(z_{k}\right)+\beta_{k} \frac{\partial \varphi_{n}\left(z_{k}\right)}{\partial t}-\frac{d z_{k}}{d t} h_{n}^{k}
$$

что с учетом равенства (3.2) можно переписать в виде

$$
\frac{\partial \psi_{n}\left(z_{k}\right)}{\partial t}=\frac{d \beta_{k}}{d t} \varphi_{n}\left(z_{k}\right)+\beta_{k} \frac{\partial \varphi_{n}\left(z_{k}\right)}{\partial t}-\frac{4 z_{k}^{2} \omega_{k}}{z_{k}^{2}-1} h_{n}^{k}, \quad k=1,2, \ldots, N .
$$

Используя формулы (3.5) и (3.21), имеем

$$
S_{n}^{0-}\left(z_{k}\right)=\frac{d \beta_{k}}{d t} \varphi_{n}\left(z_{k}\right)+\beta_{k} S_{n}^{0+}\left(z_{k}\right)-\frac{4 z_{k}^{2} \omega_{k}}{z_{k}^{2}-1} h_{n}^{k}+\sum_{m=1}^{N} g_{n}^{m}\left(p_{n}^{m-}\left(z_{k}\right)-\beta_{k} p_{n}^{m+}\left(z_{k}\right)\right) \text {. }
$$

С другой стороны, согласно формулам (2.8) и (3.9)

$$
p_{n}^{m-}\left(z_{k}\right)-\beta_{k} p_{n}^{m+}\left(z_{k}\right)=2 \beta_{k} \sum_{j=-\infty}^{\infty} f_{j}^{m} \varphi_{j}\left(z_{k}\right)
$$

Поскольку в силу леммы 1 собственные векторы, соответствующие различным собственным значениям, ортогональны, получаем

$$
\sum_{m=1}^{N} g_{n}^{m}\left(p_{n}^{m-}\left(z_{k}\right)-\beta_{k} p_{n}^{m+}\left(z_{k}\right)\right)=2 g_{n}^{k} \sum_{j=-\infty}^{\infty} f_{j}^{k} \psi_{j}\left(z_{k}\right), \quad k=1,2, \ldots, N .
$$

По определению векторов $h_{n}^{k}$

$$
h_{n}^{k}=\delta_{k} g_{n}^{k}+\gamma_{k} \psi_{n}\left(z_{k}\right) .
$$

Подставляя равенство (3.24) в (2.14), с учетом формул (1.3) и (3.12) получим

$$
\delta_{k}=-\frac{\beta_{k} c_{k}^{-}\left(z_{k}-z_{k}^{-1}\right) \dot{\alpha}\left(z_{k}\right)}{2 \omega_{k}}=-\frac{c_{k}^{+}\left(z_{k}-z_{k}^{-1}\right) \dot{\alpha}\left(z_{k}\right)}{2 \omega_{k}} .
$$

Хорошо известно, что

$$
\dot{\alpha}\left(z_{k}\right)=-\frac{1}{z_{k}} \sum_{j=-\infty}^{\infty} \varphi_{j}\left(z_{k}\right) \psi_{j}\left(z_{k}\right)
$$

поэтому на основании выражения (3.23) равенство (3.22) можно переписать в виде

$$
S_{n}^{0-}\left(z_{k}\right)-\beta_{k} S_{n}^{0+}\left(z_{k}\right)=\frac{d \beta_{k}}{d t} \varphi_{n}\left(z_{k}\right)-\frac{4 z_{k}^{2} \omega_{k}}{z_{k}^{2}-1} h_{n}^{k}-2 z_{k} c_{k}^{+} \dot{\alpha}\left(z_{k}\right) g_{n}^{k} .
$$


Следовательно, с учетом формул (3.19), (3.24) и (3.25) можно записать

$$
G_{k}=\left(\frac{d \beta_{k}}{d t}-\frac{4 z_{k}^{2} \gamma_{k} \omega_{k}}{z_{k}^{2}-1} \beta_{k}\right) \varphi_{n}\left(z_{k}\right)
$$

Сравнивая формулы (3.20) и (3.26), получим

$$
\frac{d \beta_{k}}{d t}=\left(z_{k}^{-1}-z_{k}+\frac{4 z_{k}^{2} \gamma_{k} \omega_{k}}{z_{k}^{2}-1}\right) \beta_{k}, \quad k=1,2, \ldots, N .
$$

Теперь нормировочные константы $C_{k}(t)$ находятся из равенств

$$
C_{k}^{2}(t)=-\frac{\beta_{k}(t)}{z_{k}(t) \dot{\alpha}\left(z_{k}(t), t\right)}, \quad k=1,2, \ldots, N .
$$

Итак, нами доказана следующая теорема.

Теорема. Если $a_{n}(t), b_{n}(t), f_{n}^{k}(t), g_{n}^{k}(t), k=1,2, \ldots, N, n \in \mathbb{Z}$, являются решением задачи (1.1)-(1.5), то данные рассеяния оператора

$$
L(t) y \equiv a_{n-1}(t) y_{n-1}+b_{n}(t) y_{n}+a_{n}(t) y_{n+1}
$$

определяются из равенств (3.2), (3.18) и (3.27).

Полученные равенства полностью определяют эволюцию данных рассеяния для оператора $L(t)$, что вместе с равенством (3.24) позволяет применить метод обратной задачи рассеяния для нахождения решений задачи (1.1)-(1.5).

\section{4. ЗАКЛЮЧЕНИЕ}

Из равенства (3.2) следует, что при выполнении условия (1.4) при $t>0$ выполнено неравенство $\left|\lambda_{k}(t)\right|>1$ и, следовательно, $-1<z_{k}(t)<1, k=1,2, \ldots, N$.

Заметим, что при $|z|=1$ выполнено равенство $\bar{z}=z^{-1}$. Поэтому для функции $\theta(z, t)$, введенной в равенстве $(3.18)$, получим $\bar{\theta}(z, t)=-\theta(z, t)$ при $|z|=1$. Следовательно, при любом фиксированном $t>0$, функция $\theta(z, t)$ принимает чисто мнимые значения или ее значение равно нулю, причем в точках $z= \pm 1$ она обращается в нуль. Поэтому при $|z|=1$

$$
|R(z, t)|=|R(z, 0)| \leqslant 1, \quad t>0
$$

причем знак равенства может достигаться лишь в точках $z= \pm 1$. Кроме того,

$$
\bar{R}(z, t)=R(\bar{z}, t), \quad t>0 .
$$

Значит, функция $R(z, t)$ при $t>0$ удовлетворяет условиям $(2.10),(2.11)$. Из полученного нами ранее равенства

$$
\alpha(z, t)=\alpha(z, 0) \exp \left\{2 \sum_{m=1}^{N} \int_{0}^{t}\left(\frac{1+z_{m}(\tau) z}{1-z_{m}(\tau) z}-\frac{1+z_{m}(\tau) z^{-1}}{1-z_{m}(\tau) z^{-1}}\right) \frac{\omega_{m}(\tau)}{z_{m}(\tau)-z_{m}^{-1}(\tau)} d \tau\right\}
$$


имеем

$$
\alpha(0, t)=\alpha(0,0) \exp \left\{4 \sum_{m=1}^{N} \int_{0}^{t} \frac{\omega_{m}(\tau)}{z_{m}(\tau)-z_{m}^{-1}(\tau)} d \tau\right\} .
$$

Кроме того, из равенства (3.2) получаем

$$
z_{k}(t)=z_{k}(0) \exp \left\{4 \int_{0}^{t} \frac{\omega_{k}(\tau)}{z_{k}(\tau)-z_{k}^{-1}(\tau)} d \tau\right\} .
$$

Таким образом, с учетом $|R(z, t)|=|R(z, 0)|$ мы получаем, что равенство (2.12) выполнено.

Отсюда следует (см. книгу [12]), что если начальные данные задачи (1.1) быстро убывают (т.е. $a_{n}^{0}-1 / 2$ и $b_{n}^{0}$ вместе со своими разностями всех порядков убывают при $|n| \rightarrow \infty$ быстрее любой степени $\left.|n|^{-1}\right)$, то $a_{n}(t)$ и $b_{n}(t)$ также являются быстроубывающими. Для задачи (1.1)-(1.5), рассматриваемой в данной работе, выполнение условия (1.5) при выполнении второго условия для начальных данных можно показать точно так же, как это сделано в работе [6], но с использованием результатов статьи [10].

\section{Список литературы}

[1] M. J. Ablowitz, D. J. Kaup, A. C. Newell, H. Segur, Stud. Appl. Math., 53 (1974), 249.

[2] А. Ньюэлл, "Обратное преобразование рассеяния", Солитоны, ред. Р. Буллаф, Ф. Кодри, Мир, М., 1983, 193.

[3] Ф. Калоджеро, А. Дегасперис, Спектралъные преобразования и солитонъ. Методы решения и исследования нелинейных эволючионных уравнений, Мир, М., 1985.

[4] С. В. Манаков, ЖКЭТФ, 67:2 (1974), 543.

[5] H. Flaschka, Progr. Theor. Phys., 51:3 (1974), 703.

[6] Ag. Kh. Khanmamedov, Ukrainian Math. J., 57:8 (2005), 1350.

[7] V. K. Mel'nikov, Inverse Problems, 6 (1990), 809.

[8] Г. У. Уразбоев, А. Б. Хасанов, ТМФ, 129:1 (2001), 38.

[9] K. M. Case, M. Kac, J. Math. Phys., 14 (1973), 594.

[10] Г.Ш. Гусейнов, ДАН СССР, 231:5 (1976), 1045.

[11] М. Тода, Теория нелинейных решеток, Мир, М., 1984.

[12] Л. А. Тахтаджян, Л. Д. Фаддеев, Гамильтонов подход в теории солитонов, Наука, М., 1986.

Поступила в редакцию 30.01.2007, после доработки 15.03.2007 